\title{
EL ISLAM VISTO POR EL IMPERIO BIZANTINO. LA REVELACIÓN COMO UNA HEREJÍA CRISTIANA.
}

\author{
Ma. Antonieta Emparán F. \\ Albert-Ludwigs-Universität Freiburg
}

Resumen: La revelación islámica surge en un momento en el que el cristianismo se ve a sí mismo como única religión posible tras haber superado al paganismo y encontrarse en medio de una lucha en contra de las herejías. Es por esto que el Imperio bizantino no ve como una amenaza ni como una nueva religión al Islam, sino más bien como un castigo divino y una herejía más en medio de los días del fin de los tiempos.

Palabras clave: Invasiones árabes - Apocalipsis - Islam - Herejía - Profecías.

\section{Islam seen by the Byzantine Empire. Revelation as a Christian heresy.}

\begin{abstract}
The Islamic revelation comes at a time when Christianity sees itself as the only possible religion after having overcome paganism and find itself during a struggle against heresies. Therefore, the Byzantine Empire does not see as a threat or a new religion to Islam, but rather as a divine punishment and heresy more during the end times.
\end{abstract}

Keywords: Arab invasions - Apocalypse - Islam - Heresy - Prophecy.

Recibido: 4.11.16 - Aceptado: 23.03.17

Correspondencia: María Antonieta Emparán. Email: maria.antonieta.emparan@orient.uni-freiburg.de Albert-Ludwigs-Universität Freiburg 


\section{Introducción}

a visión que los bizantinos tenían de los musulmanes quedó
registrada en variadas fuentes bizantinas. Pese al apagón
cultural, entre los siglos VII y IX, que vivió el Imperio debido a una profunda crisis que marcará un periodo intermedio en su historia (Marín, 1997), encontraremos referencias al Islam en varios textos de carácter más bien profético y/o apocalíptico. Dentro de este periodo se desarrolló una importante obra historiográfica, la cual registra gran parte de la historia del Imperio y sus interacciones con los vecinos; la Cronografia de Teófanes. Esta obra dedica un importante espacio a relatar las acciones realizadas por los musulmanes a partir del surgimiento de la revelación recibida por Muhammad. Será este texto uno de los definitivos en cuanto a la recreación de un perfil de los árabes, el Islam y Muhammad. Sin embargo, hay una importante cantidad de fuentes que abordan las invasiones musulmanas relacionadas a una interpretación apocalíptica principalmente. La más importante de estas es La Herejía de los Ismaelitas de San Juan Damasceno.

En el siguiente trabajo se aborda la visión bizantina de los árabes desde la revelación hasta el califato de Amin, sucesor de Hārūn AlRašīd. Debido a que el Islam siempre fue considerado como una herejía cristiana más, de acuerdo a las fuentes, nunca se consideró realmente una amenaza. A pesar de las invasiones y el sufrimiento que estas causaron en la población bizantina cristiana, ésta siempre la tomó como un acto de ira divina y el cumplimiento de profecías ya anunciadas, por lo que, la única vía de solución y liberación de tan terrible yugo era la permanencia en la rectitud de la fe cristiana.

\section{1.- Relación entre el cristianismo y los árabes}

El desprecio que tenían los cristianos sobre los árabes es anterior al surgimiento del islam y proviene del rechazo que sentían los pueblos sedentarios sobre los nómades. Amiano Marcelino, quien escribe a fines del siglo IV, realiza una caracterización de los árabes manifestando tal sentimiento:

The Saracens, however, whom we never found desirable either as friends or as enemies, ranging up and down the country, in a brief space of time laid waste whatever they 
could find, like rapacious hawks which, whenever they have caught sight of any prey from on high, seize it with swift swoop, and directly they have seized it make off. [...] No man ever grasps a plough-handle or cultivates a tree, none seeks a living by tilling the soil, but they rove continually over wide and extensive tracts without a home, without fixed abodes or laws; they cannot long endure the same sky, nor does the sun of a single district ever content them. Their life is always on the move, and they have mercenary wives, hired under a temporary contract. [...] Moreover, they wander so widely as long as they live, that a woman marries in one place, gives birth in another, and rears her children far away, without being allowed any opportunity for rest. They all feed upon game and an abundance of milk, which is their main sustenance, on a variety of plants, as well as on such birds as they are able to take by fowling; and I have seen many of them who were wholly unacquainted with grain and wine. So much for this dangerous tribe. (Ammianus Marcellinus, 1935, p. 27, 29 / XIV, 4)

Se los llamaba peyorativamente Ismaelitas, así como también Sarracenos identificándolos, a través de las genealogías bíblicas, como descendientes de Ismael, hijo de Abraham con Agar. Por tal motivo también se los nombraba Agarenos. De este modo, el perfil que en el Génesis se le otorga a Ismael como "...hombre fiero; su mano será contra todos, y la mano de todos contra él, y delante de todos sus hermanos habitará" (Gen 16:14) será transferido a los árabes (Fletcher, 2005, p. 23) ${ }^{1}$. La palabra sarracenos no está del todo claro su origen; por una parte podría estar

1 El término ismaelita será utilizado por los musulmanes para reforzar su relación con Abraham a partir del momento en que se distancian de los judíos en Medina tras la expulsión de las tribus judías y la aniquilación de los Qurayza, que en un primer momento fueron sus aliados. Es en este momento en el que redirigirán sus oraciones hacia la Kaba y ya no más hacia Jerusalén (Bresc et al., 2001, p. 16); Sin embargo, al parecer este término se lo habrían asignado los judíos en un tono peyorativo debido a la asociación con Ismael, del mismo modo que habrían hecho con los cristianos al relacionarlos con Edom (Durán Velasco, 2012, p. 151) 
relacionado a Sara, la esposa de Abraham, y provenir del griego sarakenos ${ }^{2}$ (Sénac, 2011, p. 20). Otra posibilidad es que provenga del árabe sarraquin, que quiere decir ladronísimos (Durán Velasco, 2012, p. 174).

Isidoro De Sevilla, contemporáneo de Muhammad, señala respecto de los árabes lo que en la época era una opinión generalizada respecto de éstos:

Saraceni dicti, vel quia ex Sarra genitos se praedicent, vel sicut gentiles aiunt, quod ex origine Syrorum sint, quasi Syriginae. Hi peramplam habitant solitudinem. Ipsi sunt et Ismaelitae, ut liber Geneseos docet, quod sint ex Ismaele. Ipsi Cedar a filio Ismaelis. Ipsi Agareni ab Agar; qui, ut diximus, perverso nomine Saraceni vocantur, qui ex Sarra se genitos gloriantur. (Isidorus Hispalensis, 1957, IX.ii.57)

Cuando surge el Islam, en el año 610, el cristianismo luchaba internamente con diversas herejías que diferían de la ortodoxia en relación a la naturaleza de Cristo. De estas herejías, será trascendental la existencia del monofisismo, la cual se encontraba ampliamente diseminada en la zona que abarca desde Armenia hasta Egipto incluyendo provincias importantes como Siria y Palestina (Fletcher, 2005, p.21).

\section{1.- Gasaníes:}

El Imperio de Oriente contrató a tribus árabes del norte para que sirvieran como tapón frente a otras confederaciones. Los árabes gasaníes, sirvieron de protectores de las fronteras imperiales que iban desde el Éufrates hasta el Sinaí. "A los jefes gasaníes se le otorgó el título de filarca, un título administrativo griego" (Kennedy, 2007, p. 32) a cambio de su trabajo. Esta relación pudo mantenerse a través de actos diplomáticos, el pago de subsidios, así como también a través de alianzas de parentesco.

2 Posibles etimologías para Sarak son: shark-el este; saraka-ladrón; y Sawarik - una tribu. Otra etimología posible para esta palabra es shrkt (federación), proveniente del griego-arameo, sin embargo, esta posibilidad ha sido desestimada como una mala interpretación de la palabra shrbt (tribu). Lo concreto es que este término aparece en los registros de Dioscórides de Anazarba y Plinio el Viejo durante el siglo I d.C. haciendo referencia a la gente que habitaba el noroeste de la Península Arábiga. Ver (Shahid \& Bosworth, 1997, págs. 27-28) para el detalle de los siguientes usos y referencias del término. 
Esta tribu prontamente se convirtió al cristianismo a través de la predica monofisita, la cual ya comenzaba a ser considerada como herética. Estos tenían un estilo de vida seminómada; los jefes se instalaban a las afueras de las ciudades, lugar al que el resto de la tribu llegaba a visitarlos. Sin embargo, debido a una posible independencia de éstos o bien a intentos de ahorrar por parte del imperio, las subvenciones cesaron, por lo que la protección de las fronteras también. Esto no solo significó el cese de la protección fronteriza, sino que también del sistema de espionaje político que realizaban dentro de las zonas que iban más allá de la frontera imperial. Una de las consecuencias de esto fue pérdida de contacto con lo que pasaba en el sur de la Península Arábiga, perdiendo todo referente de lo que vino a suceder en Makkah y Yatrib (Adamec, 2009b; Fletcher, 2005, p. 24; Kennedy, 2007, pp. 32 - 33). De este modo, la existencia de Muhammad será desconocida en el Imperio bizantino durante sus primeros tiempos.

\section{2.- Muhammad y los primeros años de expansión islámica}

Cuando Muhammad recibe la revelación coránica, la situación política en oriente se encontraba dividida en dos grandes imperios, el bizantino y el persa. Cada uno de estos se encontraba con problemas religiosos internos: El Imperio bizantino enfrentaba disputas en relación con la naturaleza de Cristo. En Egipto enviaron al exilo al patriarca Benjamín y obligaron a la apostasía a obispos, sacerdotes y monjes coptos debido al monotelismo. En Siria y Mesopotamia también ocurría algo similar; la población estaba dividida entre diferentes confesiones cristianas: la ortodoxia griega profesada solo por la aristocracia; los monofisitas reunidos en torno a la figura del patriarca de Antioquia; y los cristianos iraníes e iraquíes que en 485 establecieron un catholicos nestoriano. La consecuencia de las persecuciones a estos grupos fue el reforzamiento de las iglesias semioficiales, tal como sucedió tras la expulsión de los nestorianos por parte del emperador Zenón en 491(Bresc, Guichard, \& Mantran, 2001, pp. 10 - 11).

En cuanto al Imperio persa sasánida, aun persistían las herejías a pesar de que el pueblo se había convertido de manera obligada y aparente a la ortodoxia del zoroastrismo y los fuegos de otras ramas se apagaron (Bresc et al., 2001, p. 11). A esta situación religiosa se suma el agotamiento causada por la guerra llevada a cabo entre ambos imperios; un gran gasto de recursos para enfrentar la guerra y una merma militar significativa (Bresc et al., 2001, p. 11). 


\section{1.- Muhammad, profeta armado}

Desde que Muhammad inicia su prédica pública, comienza a realizar una profunda reforma política y social al interior del sistema tribal reinante en la península arábiga (Bramon, 2009; Bresc et al., 2001; Subhani, 1989). Una de las primeras modificaciones que impone al sistema tribal es la prohibición de asesinar a las niñas recién nacidas y de matrimonios consanguíneos. Con estas dos proscripciones Muhammad genera una ampliación demográfica $\mathrm{y}$, por otro lado, la necesidad de relacionarse familiarmente con otras tribus debido a que el matrimonio ya no se podría celebrar entre miembros de un mismo clan (Bresc et al., 2001, p. 14). Sumado a lo anterior, un primer círculo cercano al profeta, los primeros conversos, se conformó por jóvenes y débiles que se encontraban excluidos de esta sociedad tribal. Esto propició el surgimiento de los cimientos para una verdadera revolución social (Bresc et al., 2001).

Por otra parte, una de las principales consecuencias de la hégira será el establecimiento de la umma, la comunidad de fieles, lo que significó un naciente estado en expansión. Pese a este exilio, la tribu de los Qurayš continuó con sus tentativas de ataque, es por esta razón que se fomentó el saqueo de caravanas que se dirigían a Makkah (Kennedy, 2007,p. 44). Esto, por otro lado, significó una militarización de la umma, puesto que debían, primero defenderse de los Qurayš, conquistar la Kaaba, y mantenerse a través de capturas de botín que ahora se repartía entre la comunidad (Bresc et al., 2001, p. 16). Una vez finalizada la ridda ${ }^{3}$, para controlar a las tribus beduinas acostumbradas al saqueo, se las envió fuera de las fronteras de la umma liderada por la élite islámica. De esta manera, estos grupos fueron dirigidos en contra del Imperio bizantino y del Imperio sasánida. Estas campañas comenzaron antes de que la ridda acabara completamente y fue un aliciente para que las tribus se unieran a la comunidad islámica y se restablecieran los pactos de alianza bajo la promesa de entregar las mejores tierras y partes de botín de acuerdo al diwan ${ }^{4}$ establecido por Abu Bakr.

3 Una vez fallece Muhammad, todas las alianzas realizadas entre tribus se dan por anuladas. En este momento comienzan las guerras ridda (apostasía) por intentar devolver el control a Medina a través del nuevo liderazgo de Abu Bakr, quien deberá reestablecer todas las alianzas (Adamec, 2009d).

4 Para restablecer las alianzas realizadas por Muhammad, Abu Bakr puso en marcha un diwan, que era el registro de los conversos en el orden que lo hacían. Con este listado se establecían los pagos que recibirían por el botín capturado 
Sobre el combate en contra de los infieles el Qur'an señala: "Y combatid por la causa de Dios a aquellos que os combaten, pero no cometáis agresión pues, ciertamente, Dios no ama a los agresores." (Q 2:190). Esto constituye la base para el pequeño yihad ${ }^{5}$. A través de este se defiende el islam y a la contraparte se le ofrece como alternativas la conversión, el pago de impuestos o la guerra continua (Adamec, 2009c; Kennedy, 2007, p. 50). En la tradición islámica se encuentra presente la idea del premio al martirio; la entrada inmediata al paraíso si es que un fiel muere en el campo de batalla del yihad. Existen historias aisladas de musulmanes que se entregan voluntariamente a la muerte en el campo de batalla, sin embargo, lo frecuente es que quisieran disfrutar de los beneficios terrenales tras la repartición del botín de guerra (Kennedy, 2007, p. 65).

Los Omeya fueron los encargados de las primeras expediciones invasivas en contra del Imperio bizantino lideradas por Muawiya, Jalid y Amr, durante los califatos de Umar, Uthman y el Omeya (Bresc et al., 2001, p. 22). La tradición militar Omeya explicaría en gran parte el formidable éxito que estos tres comandantes tendrán en sus expediciones y en la administración de sus respectivas provincias posteriormente.

\section{2.- Islam, otra herejía cristiana}

En el momento en que el Islam surge, el cristianismo ya se había apoderado de todo el ámbito religioso, intelectual y filosófico del mundo romano. El mundo pagano de la antigüedad ya había quedado en el olvido a través de diversas prácticas de debilitamiento de estos modelos. Si bien el mundo pagano aún no había sido extinguido por completo y perduraban los zoroastrianos en Persia, adoradores de árboles y fuentes en el Mediterráneo, se esperaba que ellos pronto encontraran la palabra de Dios y reconocieran

\section{(Adamec, 2009a).}

5 Etimológicamente la palabra yihad quiere decir esfuerzo. Este esfuerzo se refiere al que se realiza por mantener la religiosidad. Existen dos tipos de yihad: Gran yihad, el cual es un esfuerzo espiritual que cada individuo lleva a cabo con el fin de mejorar su islam. El Pequeño yihad es, por otra parte, de carácter físico y está relacionado directamente con la guerra. Este yihad, sin embargo, está prescrito solo en caso defensivo y el Corán autoriza la actitud combativa en caso de ser objeto de alguna injusticia. El Corán además norma la guerra defensiva estableciendo ciertas reglas para el desarrollo de la guerra (Melo, 2006; Tyan, 1991). 
a Cristo. A los judíos se los reconocía en tanto tales y, ante el rechazo de la verdadera fe, no se esperaba otra cosa que pagaran por esto (Fletcher, 2005 , pp. 30 - 31). Por lo tanto, el Islam surge en medio de un mundo en el que el cristianismo aparece como la única posibilidad de salvación y el único camino religioso y/o filosófico posible.

Tras la muerte de Abu Talib, el tío de Muhammad, las persecuciones se hicieron cada vez mayores en contra de los musulmanes. El profeta, ante esta situación, decide enviar a un pequeño grupo al reino cristiano de Abisinia el año $615^{6}$ (Bresc et al., 2001, p. 14; Subhani, 1989, p. 104). Este hecho, en conjunto a los pasajes coránicos dedicados a la imagen de la Virgen María, así como el hadit del momento en que Muhammad entra a la Kaaba y destruye todos los ídolos y protegiendo tan solo una imagen de la Virgen con el niño Jesús, mostrarán unos fuertes lazos con el cristianismo.

Quien será determinante para establecer al islam como otra herejía del cristianismo es San Juan Damasceno ${ }^{7}$, quien analiza el nuevo fenómeno religioso en La Herejía de los Ismaelitas. En ésta, como en todas las obras en la que hace mención de ellos, no los considerará nunca como una nueva religión, sino que señala que tal como el arrianismo, el Islam es una nueva herejía basada en un falso profeta que ha construido su fundamento teológico gracias a la lectura del Antiguo y Nuevo Testamento, así como la cercanía que tuvo con un arriano. Damasceno realiza una crítica al Islam no solo en la Herejía de los Ismaelitas, sino que a través de diálogos va señalando los errores que comete esta nueva herejía en relación a la ortodoxia (Janosik, 2003).

\section{3.- Apocalíptica}

En un principio, la entrada de los musulmanes no fue recibida por lo bizantinos bajo un carácter apocalíptico según señala Jean Flori. Los motivos son dobles: en el mundo cristiano no existía unanimidad respecto de la naturaleza cristológica. Es por esta razón que en las zonas invadidas por los árabes existía una gran presencia de lo que la ortodoxia llamó herejías y persiguió. Entre estas, además de la monofisita, se encontraban grupos nestorianos, siríacos, armenios, monotelitas, etc. Con la llegada de los árabes, estos grupos se vieron liberados de las persecuciones bizantinas, mientras que los árabes musulmanes no distinguían las sutiles diferencias

6 Esta será considerada la primera hégira, que solo realizará un pequeño grupo.

7 Cfr. Infra.

162 
entre un cristianismo y otro. Por otra parte, la situación política del lugar ya estaba bastante inestable debido a las guerras con Persia. Por lo tanto, cuando llegan los árabes no se encontraron con mayores resistencias (Flori, 2010, pp. 98 - 99). Sin embargo, a pesar de la relativa facilidad de invasión, esta no estuvo vacía de los horrores tradicionales de cualquier guerra. Es por esto que, a partir del natural juicio desfavorable por parte de los vencidos, se construyera una imagen barbárica de los árabes musulmanes apoyada principalmente por la idea que ya se tenía de ellos desde tiempos anteriores (Flori, 2010, p. 99).

La asociación que el Imperio bizantino hace entre los musulmanes e ideas apocalípticas la podemos dividir en tres momentos: El primer periodo de invasiones y éxito musulmán; Un segundo periodo de asentamiento e interpretaciones proféticas; Y, un tercer momento de estabilidad musulmana en el que surgen nuevas profecías.

\section{1.- Invasiones árabes}

3.1.1.- Patriarca Sofronio de Jerusalén:

Sofronio, el patriarca de Jerusalén al momento en que llegan los árabes es quien tendrá que entregar la capitulación de la ciudad a Umar en 638. Éste, en una carta sinodal escrita a comienzos del año 634, señala que :

My intention is that God himself [...] when he has appeased by Your prayers which are acceptable to God [...] provide them [the Christ-loving] with strong and mighty authority over all barbarians but especially the Saracens, destroying their pride. Through our sins they [sc. the Saracens] have now unexpectedly risen up against us, and are carrying everything off as booty with cruel and savage intent and impious and godless daring. (Sophronius, 2009, pp. 153, 155)

En este pasaje se puede ver la perspectiva del patriarca respecto de los árabes musulmanes; estos se muestran ya como castigadores de los propios pecados de la cristiandad bizantina, es por esto que los sarracenos se levantan inadvertidamente ${ }^{8}$ (Fletcher, 2005, p. 29; Flori, 2010, pp. 99 -

8 Se puede relacionar esta inadvertencia por parte del Imperio bizantino al cese 
100, 105 - 107).

Sin embargo, las milicias imperiales no han podido terminar con "el orgullo de todos los bárbaros", sino que la ambición de estos va más allá queriendo someter a su propia ley toda esta zona como señala Flori. Respecto de esta poderosa invasión, el patriarca señala en su Sermón sobre la Teofanía durante el verano del mismo año lo siguiente:

¿Por qué razón las incursiones bárbaras se multiplican y las falanges sarracenas se han levantado contra nosotros? [...] Los sarracenos recorren comarcas que le han sido prohibidas, saquean ciudades, asolan los campos, entregan las aldeas a las llamas, derriban los sagrados monasterios, plantan cara a los ejércitos romanos, consiguen trofeos en la guerra, añaden victoria sobre victoria, se alinean en masa contra nosotros [...] y se vanaglorian de conquistar el mundo entero" (Flori, 2010, p. 100)

Sofronio atestigua la fuerza con la que llegan los árabes musulmanes invadiendo tierras cristianas, específicamente Jerusalén y sus alrededores y la destrucción de iglesias y monasterios. Debido a la ocupación de los lugares sagrados para el cristianismo por parte de los musulmanes, la misa de Navidad del año 634 no se pudo realizar como de costumbre en el templo de Belén. Por lo que el Patriarca celebró la misa en la basílica de Santa María (Cosma, 1940, p. 77). Sin embargo, éste insistía en que la solución se centraba en el arrepentimiento de los propios cristianos para su pronta desaparición y en su sermón navideño señaló: "A causa de vuestros numerosos pecados todos debemos permanecer en casa, no porque estemos atrapados, sino por miedo a los Sarracenos. Pero si fuéramos dignos, podríamos ir a todos los lugares cercanos y rezar y cantar 'Alabado sea el Señor y la paz en la tierra'...” (Sophonius, 1886, 146r, 27-6)

Sofronio pudo contemplar los trabajos de construcción de la mezquita de Umar en la explanada del Templo de Salomón, para los que se contrataron cristianos. El patriarca interpreta pasajes bíblicos en los que señala la ocupación de Jerusalén como la abominación de la desolación a la invasión árabe, pese a que hay pasajes en la biblia en los que señala

del flujo de información respecto de la Península Arábiga que antes entregaban los Gasaníes. 
claramente a romanos y griegos como estos invasores ${ }^{9}$.

El Patriarca nunca identifica a los sarracenos con una nueva religión, sino que los ve permanentemente como una herramienta de Dios para castigar a los cristianos por sus propios pecados. Éste veía a los árabes musulmanes como gentes que bien no creían en Dios o bien lo odiaban. Como ya fue dicho, la estrategia para derrotarlos estaba en el arrepentimiento de los pecados cometidos y el retorno a la ortodoxia; el armamento militar y el contragolpe en la batalla no era la vía de solución a este problema (Kennedy, 2007, p. 417).

\subsection{2.- Máximo el confesor:}

Seis años más tarde, Máximo el Confesor, teólogo ortodoxo, señala que el horror provocado por los bárbaros se ha logrado gracias a la compañía de los judíos ya que son los precursores del anticristo. Así, el teólogo señala que la alianza entre los judíos y Muhammad está realizada bien bajo una alianza voluntaria o bajo sometimiento (Flori, 2010, pp. 101, 105). En una carta enviada a Petrus Illustris, exarca imperial de la provincia de África, entre los años 643 o 644 en contra del monofisismo, Máximo el Confesor señala en relación con los problemas al interior de la iglesia y de la llegada de los musulmanes denominados como bárbaros lo siguiente:

En effet le divin Sophrone, comme vous en êtes témoins, vous très louables, y faisait obstacle dans sa région d'Afrique, avec moi et tous les moines étrangers, lorsque eux combinaient de renverser la foi à la face de tous... [...] Mais eux qui jalousaient leur accord et leur confession du Christ-Dieu, aussitôt, tel un nuage d'obscurité, tel le flot strident et écrasant de la mer et des Barbares, sans que nul les remuât que le seul démon ami des turbulences qui excite à la haine du genre humain, ont imaginé l'Ekthèse contre la vraie foi des chrétiens; à sa place ensuite ce

9 Principalmente vemos en los libros de Daniel, Marcos y Lucas en los que se señala el tema de la abominación de la desolación sin señalar grupo alguno al que se adjudica la invasión. Sin embargo, vemos libros como Juan y Hechos en los que se indica como los invasores responsables de la abominación de la desolación a romanos y griegos. Cfr. Daniel 11:31; Marcos 13:14; Lucas 21:2022; Hechos 21:28; y Juan 11:46-48. 
furent des assertions, des systèmes [pour gens] incultes; ensuite des actions pour les rappeler; ensuite des conciles de brigandage et des concours d'évêques, non pas tant rassemblés que forcés à le faire; non pas tant expédiant les choses que partant à l'étranger en fuyant les Barbares; ensuite des ordres impératifs, des menaces portées ici et là contre les fidèles. [...] Aussi bien, qui et quel Sophrone, homme si connu, a persuadé ces menteurs de faire de telles abominations? Quelle Église ne les a-t-elle mises en question? Quel guide pieux et orthodoxe ne les a suppliés en les conjurant sur tous les tons de mettre un terme à leur hérésie? (Maxime le Confesseur, 1998, pp.187-188)

\subsection{3.- Doctrina Jacobi Nuper Baptizati}

Entre los años 630 y 640 un judío recién converso al cristianismo escribe una apología antijudía en la que señala también el carácter falso del profeta de los sarracenos. Uno de los principales elementos que lo lleva a negar el carácter profético del líder de los árabes es que este va completamente armado y su pueblo se levanta violentamente en contra de las zonas que está invadiendo (Fletcher, 2005, p. 29; Flori, 2010, p. 101). En la introducción de la publicación de 1910 Bonwetsch resume:

Nach Theophanes wurde auch Omar von jüdischer Seite angereizt, gegen die Heiligtümer der Christen vorzugehen. Am wertvollsten unter den zeitgeschichtlichen Mitteilungen sind wohl die über das Vordringen der Saracenen unter Mahammed. Es ist bekannt, wie die Juden Muhammeds Auftreten erwartungsvoll begrüßten. Unser Dialog illustriert diese Tatsache. Er setzt jedoch voraus, daß Muhammed noch lebte, als Sergius gegen die Saracenen fiel. Eigentümlicher Weise wird die Zeit der Knechtschaft der Juden auf 640, dagegen auf 600 Jahre angegeben; es dürfte von einem verschiedenen Zeitpunkt aus gerechnet sein. V, 20 S.91 wird der Dialog auf den 13. Juli des 7. Jahrs des Indiktions datiert, unter Kaiser Heraklius, also auf das Jahr 634. (Bonwetsch, 1910, pp. XV-XVI) ${ }^{10}$

10 Ver los pasajes V, 16 S. 86; I, 22 S. 21,7 y II, 6 S. 49, 1 del texto original en latín. 


\subsection{4.- Juan de Nikiou}

El cronista Juan de Nikiou, monofisita copto, escribe su obra hacia el 690. En su crónica deja en claro que los verdaderos malos de la época son los Sasánidas. El problema real, en relación a las invasiones sarracenas, sigue estando en la desviación respecto a la naturaleza de Cristo. En este caso el castigo está dirigido a quienes han dividido la naturaleza del encarnado (Flori, 2010, pp. 102-103; Kennedy, 2007, p. 425):

When God's judgement lights upon these Ishmaelites may He do unto them as He did aforetime unto Pharaoh! But it is because of our sins that He has suffered them to deal thus with us. Yet in His longsuffering our God and Saviour Jesus Christ will look upon us and protect us: and we also trust that He will destroy the enemies of the Cross, saith the book which lies not.

And all these things fell out because they divided Christ into two natures, whilst some of them made Him (merely) a created being. Also the Roman emperors lost the imperial crown, and the Ishmaelites and Chuzaeans won the mastery over them, because they did not walk in the orthodox faith of our Lord Jesus Christ, but divided the indivisible. (Nikiu, 1982, CXX.33 y CXXIII.4-5)

A pesar de que retrata mucho mejor a los árabes que a los persas, es muy crítico con estos y también lo es con quienes los han ayudado. Señala el yugo al que han sido sometidos por parte de Amr, quien les obliga a pagar un alto impuesto y que quienes no tienen para pagar han de esconderse. Pero insiste en que, tal como aparece en la biblia, los ismaelitas serán castigados posteriormente hayan sido empleados como "viles esclavos"(Flori, 2010, p. 103) para castigar a los cristianos desviados (Kennedy, 2007, p. 425).

\section{2.- Asentamiento}

Cuando el asedio musulmán se convierte en capitulación y adición de nuevas provincias al territorio islámico, la población cristiana ve que los sarracenos no se irán tan rápido como esperaban. Es por este motivo que comienzan a hacer nuevas interpretaciones apocalípticas incorporando a los árabes dentro de estas con un rol que anuncia la llegada de los últimos días. 


\subsection{1.- Sebeos}

Este historiador armenio, a quien se le adjudica el texto ${ }^{11}$ escrito a finales del siglo VII, realiza una reinterpretación de la profecía de Daniel, reconfigurando el establecimiento de los cuatro imperios que luego darían paso a diez reinos antes del fin de los tiempos. A pesar de que el libro de Daniel establece que los cuatro imperios son el Babilónico, Persa, Griego y Romano, Sebeos señala que los imperios son el griego, persa sasánida, los pueblos de Gog y Magog y, finalmente, el reino de Ismael que se levantara desde el sur. Después vendrán los diez reyes que son los diez cuernos de la bestia para dar paso al fin de los tiempos (Howard-Johnston, 1999, pp. 1-li; Flori, 2010, pp. 111 - 113). Respecto de la profecía de Daniel Sebeos señala:

Who could describe the fearful calamity of the Ismaelite brigand who set fire to sea and land? However, the blessed Daniel had earlier prophesied such a disaster which befell the land. Through four beasts he indicated the four kingdoms which would arise on earth. [...] 'The fourth beast was fearful and amazing, and its teeth were of iron, and its claws of bronze. It ate and broke in pieces, and crushed the remnants under foot.' This fourth, arising from the south, is the kingdom of Ismael, just as the archangel explained: 'The fourth beast, the fourth kingdom, shall arise, which shall be greater than all [other] kingdoms; and it will consume the whole earth.' 'And the ten horns, the ten kings, shall arise. And after them will rise up another who is greater in evil than all the previous ones'; and what follows in order. (Sebeos, 1999, pp. 105-106)

Como se puede ver a través del texto de Sebeos, hay una percepción del poder de los árabes; los señala como el reino más poderoso de los cuatro. Por otra parte, en este relato ya vemos que los árabes cumplen un papel fundamental en el fin de los tiempos. La idea de que los males que traen consigo estas invasiones acabarán pronto sigue presente, con la diferencia de que lo que se acaba no solo es el sufrimiento que estos provocan y la dominación misma que estos ejercen, sino que lo que se

11 Historia de Heraclio. 
acaba es el tiempo. Por lo tanto, la única forma de enfrentar esta situación para los cristianos del imperio bizantino es mantenerse firme en la fe cristiana y en la ortodoxia; la única solución es mantenerse en la vía recta y evitar la herejía. Más tarde su sucesor Lewond realizará una narración de los hechos alejada de interpretaciones apocalípticas a pesar de que para la primera parte de su historia toma como referente la Historia de Heraclio (Lewond, 1982).

\subsection{2.- San Juan Damasceno}

Juan Damasceno, considerado actualmente como uno de los padres de la iglesia, nació en Siria, cuando esta provincia ya estaba en dominio islámico, probablemente el año $652^{12}$. Su abuelo, Mansur Ibn Sargun, desempeñó un importante rol en la entrega de la capitulación de la provincia a los musulmanes tras la derrota bizantina en la Batalla de Yarmuk en 636, y la promesa del general Jalid de otorgar seguridad a los habitantes de la ciudad a cambio del pago de impuestos. Mansur, por lo tanto, jugó un rol importante en la negociación con los árabes invasores, ya que desempeñaba un cargo de importancia para el Imperio Bizantino. Este mismo cargo lo siguió desempeñando para los musulmanes bajo el califato de Umar. Posteriormente lo desempeñará el padre de Juan Damasceno, Ibn Mansur y luego el propio Juan Damasceno bajo los respectivos califas (Rhodes, 2007, p. 16).

Juan Damasceno recibió una educación islámica, probablemente compartiendo la enseñanza con el príncipe Yazid I. En aquel periodo, Juan pudo estudiar la teología islámica, el Corán, el hadith y algunos poemas árabes. Sin embargo, a partir de los 12 años de edad recibirá educación griega por parte del monje Cosmas bajo el permiso del califa solicitado por Ibn Mansur (Rhodes, 2007, pp. 16-17). Es gracias a esta educación que Juan Damasceno escribe La Herejía de los Ismaelitas, en la que, como ya mencionamos, retrata a los musulmanes como una herejía cristiana

12 D. Bryan Rhodes en su tesis señala que es probable que este sea el año de su nacimiento debido al cálculo que realiza en relación a la llegada del monje Cosmas, quien será el tutor de Juan Damasceno. Este cálculo lo hace basado en la fecha en que toman como esclavos a los monjes sicilianos el año 664. Sin embargo, el autor indica que otra fecha probable es en 675 , mientras que lo más usual es fijarla entre 655 y 660 (Rhodes, 2007, p. 16). Por otra parte, Jean Flori fija como fecha de su nacimiento el año 650 (Flori, 2010, p. 113). 
sin llegar a reconocer al islam como a una nueva religión. Al respect, Damasceno señala lo siguiente: "From that time to the present, a false prophet named Mohammed $(\mathrm{M} \alpha \mu \varepsilon \delta)$ has appeared in their midst. This man, after having chanced upon the Old and New Testaments and likewise it seems having conversed with an Arian monk, devised his own heresy." (Rhodes, 2007, p. 56). Podemos ver que para Damasceno Muhammad ha construido su propia herejía cristiana con elementos del Viejo y el Nuevo Testamento, así como con elementos del arrianismo. Juan Damasceno conoce bien la versión de Cristo que tienen los musulmanes, sabe que lo consideran como un profeta enviado por Dios, pero que no reconocen en él una dimensión divina. Sin embargo, creen en la concepción milagrosa y en la virginidad de María, así como también que Dios evita que Jesús muera y sea crucificado llevándolo a los cielos (Damasceno, 1958, 153-154).

Damasceno señala que tal herejía es la fuente del apocalipsis, del que se referirá, indirectamente, en su obra Exposición de la fe ortodoxa, en el que señala que llegará el anticristo, que no es la encarnación del Diablo, sino que viene con toda su energía y se sentará en el Templo de Dios (Flori, 2010, p. 114), seguramente refiriéndose a la Cúpula de la Roca y la Mezquita al-Aqsa. Sobre esto, en la Herejía de los Ismaelitas señala: "There is also the superstition of the Ishmaelites which to this day prevails and keeps people in error, being a forerunner of the Antichrist" (Damasceno, 1958, p. 153).

\section{3.- Estabilidad}

Luego de que los árabes invadieran las provincias bizantinas, se apoderaran y establecieran firmemente en ellas, las esperanzas de una pronta retirada se van extinguiendo junto con el fortalecimiento de los nuevos gobernantes. La idea de que conservar la fe firme y la rectitud ortodoxa serían la vía para que los sarracenos, instrumento de la ira divina en contra de la herejía, se fuesen pronto comienza a extinguirse dando paso ya no solo a interpretaciones del apocalipsis, sino que a la creación de nuevos textos proféticos. Estos textos, realizados a fines del siglo VII y comienzos del VIII, son escritos en formula profética señalando los hechos que acontecerán en el fin de los tiempos, cuando en realidad es una narración de los hechos ya acontecidos con una profunda visión apocalíptica y desesperanza respecto del devenir de los tiempos. Los principales textos de este género son los de Pseudo-Metodio y Pseudo-Atanasio. 


\subsection{1.- Pseudo-Metodio ${ }^{13}$}

El texto de Pseudo-Metodio es un apocalipsis datado a fines del siglo VII que, según el mismo texto, afirma haber recibido la revelación en la montaña de Senegar. En éste señala que la aparición del Islam cumple un rol fundamental en el apocalipsis. El texto va realizando un breve resumen de los principales acontecimientos durante los siete milenios hasta llegar al punto que nos interesa; el rol que cumplen los musulmanes señalando el apocalipsis: "For in the last millennium, that is the seventh, in that time the kingdom of the Persians will be uprooted, and in that time the seed of Ishmael will come out of the desert of Yathrib and having come out they will be gathered together in one accord at Gabaoth ${ }^{14}$ the Great" (Methodius, 2012, p. 37).

A partir de este momento Pseudo-Metodio va describiendo todos los hechos ocurridos en relación con la invasión musulmana sobre el Imperio bizantino y persa; bajo la óptica apocalíptica va relatando y enunciándolos como acontecimientos que sucederán en el futuro.

And after the tribulation under the Ishmaelites has come about, when men will be in peril and afflicted, having no hope of salvation or redemption out of the hands of the Ishmaelites, hunted, afflicted, and tormented with hunger and thirst and nakedness, these barbarians will be eating and drinking and boasting of their victories and of the desolations to which they have reduced Persia and Romania, Cilicia and Syria, Cappadocia and Isauria and Africa and Sicily and those living near Rome and the islands, dressing up like bridegrooms, and blaspheming they say, "The Christians have no rescue from out of our hands "(Methodius, 2012, p. 55).

13 Este texto escrito en Mesopotamia ha sido atribuido al obispo Metodio de Olimpia quien murió el año 311. Sin embargo, este texto fue redactado entre los años 690 - 692, siendo su autor contemporáneo a los hechos que narra marcando el inicio del fin de los tiempos (Flori, 2010, p. 116; Methodius, 2012, p. vii; Ubierna, 2000, p. 475).

14 Probablemente se refiere a la batalla de Yarmuk en la que obtienen la victoria los musulmanes y marca el comienzo de la derrota a manos de éstos del Imperio bizantino (Methodius, 2012, p. 340). 
Luego de esto, vendrá el emperador bizantino y liberará a los cristianos del yugo de los ismaelitas cerca del año 700 (Flori, 2010, p. 122). Esto sostiene el mito del último emperador (Ubierna, 2000), previo a los postreros acontecimientos del fin del mundo. A la espera de que llegue el emperador que vencerá a los árabes, los cristianos deben permanecer fieles a la religión, por lo que constituye un documento tanto religioso como político (Flori, 2010, pp. 121-122); se puede ver en éste la intención de evitar que más cristianos se conviertan al islam dadas las conveniencias que esto significa, sobre todo en términos económicos ${ }^{15}$.

\subsection{2.- Pseudo-Atanasio ${ }^{16}$}

Al contrario de lo que indica el Pseudo-Metodio, en el apocalipsis del Pseudo-Atanasio escrito a comienzos del siglo VIII no señala la liberación del dominio musulmán para los cristianos, sino que revela que la llegada de los musulmanes es tan solo el inicio del resto de los desastres que devienen con el fin de los tiempos. Al igual que en el resto de las interpretaciones apocalípticas respecto de la llegada de los musulmanes, esta se debe a la depravación del clero ${ }^{17}$ y ese es el motivo por el cual arrasan monasterios. Advierte, además, de que una vez llegue Cristo nuevamente, éste se vengará de todos aquellos herejes que hayan colaborado con los musulmanes y sean responsables de la llegada de estos por sus pecados (Flori, 2010, pp. 123-124).

So now, O bishops, priests and deacons who listen to me today Instruct your people well in the fear of the Lord. Do not be negligent by not instructing them in the Holy Scriptures, for you will be responsible for their souls at God's tribunal. Do not abandon the ship by not guiding

15 Recordemos que los dimmies, las gentes del libro, cristianos y judíos debían pagar un impuesto específico para mantener la práctica de su culto. Este impuesto no debían pagarlo los musulmanes, por lo que muchos escogieron la apostasía para aliviar la carga económica.

16 Este texto está atribuido a Atanasio de Alejandría quien murió en 373. El texto fue escrito alrededor del año 700 (Flori, 2010, p. 123; Martínez, 1985, p. 248)

17 En el caso de este texto, las críticas que hace al clero no solo están dirigidas a una desviación teológica hacia una herejía, sino que acusa directamente de cometer incesto y adulterio entre otros pecados (Flori, 2010; Martínez, 1985). 
it, and it sinks. Do not relax even once by not awakening the people. Lest they perish in sin, and their sentence come upon you. For it is you who are called "shepherds of the people”: Tend your flock.(Martínez, 1985, p. 472)

Este apocalipsis tiene como fin corregir el comportamiento del clero y el de servir como guía para la conducción de los fieles incentivando que mantengan su rectitud en la fe cristiana advirtiendo de las consecuencias en el juicio final si es que no mantienen firme su religiosidad.

\section{4.- Teófanes: sobre la revelación y las conquistas}

Una vez se recibe la primera revelación coránica, la expansión del islam será vertiginosa y muy exitosa. Rápidamente comienza a expandirse fuera de la Península Arábiga y tan solo en veinte años después de la muerte de Muhammad ya habían conseguido arrebatar al imperio bizantino las provincias de Palestina, Siria y Egipto. El relato de estas invasiones, así como también los periodos de gobierno de cada uno de los califas, los registra Teófanes en su Cronografía cuya escritura fue finalizada en el año 815.

El relato de los árabes comienza en el año 6119 (626/7) señalando a Muhammad tan solo como líder de los árabes. Pasarán cuatro años antes de que los vuelva a mencionar; en 6122 (629/30) señala nuevamente a Muhammad como líder de los árabes y hace un relato del perfil del profeta y de cómo es que éste recibe la revelación. También menciona la creencia del martirio en la guerra defensiva del islam y el premio del paraíso por esto. Luego, conforme va avanzando en su cronografía, Teófanes nos va informando de los principales hechos con relación a las incursiones e invasiones árabes, la sucesión de califas y diversos acuerdos y pactos de paz que se establecen con el emperador bizantino.

En primera instancia, es preciso detenernos en la imagen que recrea Teófanes sobre Muhammad. Primero nos señala la genealogía de este hombre indicando que pertenece a la tribu descendiente de Ismael, hijo de Abraham. Señala que queda huérfano de niño y que entra al servicio de una mujer muy rica ${ }^{18}$ y nos da a entender que por interés se casa con ella, al a cuál engaña con su profecía. Sobre la revelación, Teófanes señala:

18 La mujer de la que habla es Jadiya, la primera esposa de Muhammad que, efectivamente era una viuda muy rica en La Meca. 
"Whenever he came to Palestine ha consorted with Jews and Christians and sought from them certain scriptural matters. He was also afflicted with epilepsy" (Teófanes, 1982, p. 464). Luego, Teófanes se refiere a la ida al paraíso tras la muerte en batalla de los musulmanes y las cosas que pueden encontrar los mártires ${ }^{19}$; "and other think full profligacy and stupidity; also that men should feel sympathy for one another and help those who are wronged.'(Teófanes, 1982, p. 465)

En esta descripción vemos que para Teofanes el Islam sigue siendo una herejía puesto que es una interpretación de las escrituras tanto judías como cristianas. De modo que, sumado al hecho de que es una herejía judeocristiana, esta fue diseñada por un hombre oportunista, mentiroso y aquejado por la epilepsia. Por lo tanto, la profecía no sería tal, sino que una interpretación de los textos cristianos realizada con fines oportunistas.

El año 6127 (634/5) sitúa la invasión a Palestina y el inicio de los trabajos de construcción de la mezquita sobre la explanada en la que se encontraba el Templo de Salomón. Cita a Sofronio respecto a la "abominación de la desolación", así como también el acuerdo al que éste llega con Umar en relación a la protección de los cristianos (Teófanes, 1982, p. 471). El año 6135 (642/3) comienza la construcción del templo en Jerusalén, sin embargo, la primera estructura cae y este será el motivo por el cual comienzan a retirar las cruces ${ }^{20}$.

Entre los años 6129 (636/7) y 6147 (654/5), el año en que muere Uthman, menciona 9 veces a Muawiya, quien será el principal protagonista de las invasiones musulmanas. El año 6148 (655/6) Teófanes relata la disputa por la sucesión al califato entre Muawiya y Ali señalando la victoria del primero y la consecuente obtención del califato ${ }^{21}$. En lo que seguirá,

19 Comida y bebida carnal, tener relaciones sexuales con mujeres. Hay ríos de miel, vino y leche (Teófanes, 1982, p. 465).

20 Teófanes señala que al preguntar Umar la razón por la cual se cae la estructura, los judíos le responden que es a causa de la cruz que se encuentra en la iglesia en el Monte de los Olivos y le advierten que, si no la retiran, la estructura del templo nunca quedará firme. Este será el motivo por el cual las cruces comenzarán a ser bajadas (Teófanes, 1982, p. 476).

21 Teófanes y posteriormente Constantino considerarán a Muawiya como el cuarto califa en sucesión tras la muerte de Muhammad. Conocen la existencia de Ali, pero no le conceden el título de Califa. Sin embargo, Teófanes señala que a partir de la muerte de Ali en el año 6151 (658/9) será Muawiya el único líder de 
Teófanes va relatando las principales incursiones árabes, la captura de tierras, batallas, conflictos internos y la sucesión de califas. El retrato de los musulmanes está contenido en los pasajes que acabamos de señalar. En lo que sigue no habrá una imagen tan poderosa como la de Muhammad y los cuatro califas que le siguen ${ }^{22}$.

\section{Conclusión}

Desde las primeras invasiones árabes al imperio bizantino hasta la Cronografía de Teófanes, el islam siempre aparece como una herejía cristiana y Muhammad como un falso profeta y/o blasfemo. Es por esta razón que la única solución para el mal que significaba la invasión árabe consistía en la fidelidad a la verdad cristiana, ya sea desde una perspectiva ortodoxa o monofisita. Algunas de las interpretaciones apocalípticas declaran la inutilidad del despliegue de armas en contra de los árabes, pero señala que, gracias a la fe y la destrucción de las herejías, el castigo divino desaparecerá provocando la caída de los árabes por medio de su propia mano. Teófanes, quien principalmente reitera el hecho de que el islam no es más que una herejía cristiana, menciona las disputas internas en el Imperio Islámico como consecuencia de la malicia de estos.

Podemos concluir que la verdadera amenaza, desde el comienzo de las invasiones árabes para el Imperio bizantino, era realmente la desviación de la fe, la emergencia de herejías, el comportamiento indebido del clero y otras actitudes fuera de la rectitud cristiana y las invasiones árabes solo una consecuencia de ellas. Debemos recordar que justamente las provincias capturadas por los musulmanes son las que albergaban la mayor cantidad

los árabes. Nos detenemos en este hecho precisamente por la importancia que Muawiya tiene para el imperio Bizantino, quien es el principal responsable por la captura de importantes provincias bizantinas. Teófanes continuará su cronografía mencionando el actuar de Muawiya. Podemos decir, que entre los años 6129 y el 6171 este será el protagonista de las crónicas de los árabes. Sin duda, podemos asociar este hecho no solo al hecho de que fuera el gobernador de Siria en un comienzo y luego el califa, sino que al retrato que Juan Bar Penkaye hace de éste en el que demuestra una gran admiración a él. Teófanes, a pesar de las acciones en contra del imperio que Muawiya comete, realiza una construcción positiva de éste en términos de su capacidad militar, organizativa y de liderazgo; es el hombre fuerte de la primera etapa del islam para el Imperio Bizantino. Sobre Juan Bar Penkaye: Kennedy, 2007, pp. 421-422; Ubierna, 2000, pp. 484-485.

22 Considerando a Muawiya como el cuarto califa. 
de las herejías cristianas, lo cual, de seguro, influyó para la realización de estas interpretaciones apocalípticas, que se desarrollaban, no lo olvidemos, justo en el momento en el que debe llegar el fin de los tiempos.

\section{Referencias bibliográficas}

\section{Fuentes primarias:}

AMMIANUS MARCELLINUS (1935). The Surviving Books of the History of Ammianus Marcellinus. (Trad. J. C. Rolfe). Harvard Univ. Press.: London: Heinemann / Cambridge, Mass.

DAMASCEnO, J. (1958). The Fathers of the Church, vol 37. Saint John of Damascus writings. (F. H. Chase, Trans.). Editorial Board: New York.

ISIDORUS HISPALENSIS (1957). Etymologiarum Sive Oricinum (W. M. Lindsay, Brevique anotatione critica instruxit). Oxonii: Oxford.

JACOBUS (1910). Doctrina Iacobi nuper baptizati. (Introd. G. N. Bonwetsch). Weidmann: Berlin.

JOHN OF NIKIU (1982). The Chronicle of John (c. 690 A.D.) Coptic Bishop of Nikiu. (Trad. y notas R. H. Charles). APA - Philo Press: Amsterdam.

LEWOND (1982). History of Lewond. The Eminent Vardapet of the Armenians. (Trad. intro. y com. Z. Arzoumanian). St. Sahag and St. Mesrob Armenian Church: Wynnewood.

METHODIUS, P. (2012). Apocalypse Pseudo-Methodius. An Alexrandrian World Chronicle. (B. Garstad, Ed. \& Trans.). Dumbarton Oaks: Cambridge.

MAXIME LE CONFESSEUR (1998). Opuscules Théologiques et Polémiques. (Intro. J. C. Larchet; Trad. E. Ponsoye). Les Éditions du Cerf: Paris.

SEBEOS, (1999). The Armenian History attributed to Sebeos. (trad y notas, R.W. Thomson; Comentario Histórico, J. Howard-Johnston; Asistencia, T. Greenwood). Liverpool: Liverpool University Press.

SOPHONIUS (2009). Synodical Letter. In Sophonius of Jerusalem and seventhCentury Heresy. The Synodical Letter and Others Documents. (ed. Y trad. P. Allen). Oxford University Press: Oxford.

SOPHRONIUS (1886). Weihnachtspredigt des Sophronios. (trad. al alemán y notas H. Usener) Rheinisches Museum für Philologie, 41, 500-516.

TEÓFANES. (1982). The Chronicle of Teophanes. (H. Turttedove, Trans.). University of Pensilvania Press: Philadelphia. 


\section{Fuentes críticas:}

ADAMEC, L. W. (2009a). Diwan. In Historical Dictionary of Islam $\left(2^{\circ}\right.$ ed., p. 85). The Scarecrow Press, Inc.: Maryland.

ADAMEC, L. W. (2009b). GHASSANIDS. In J. Woronoff (Ed.), Historical Dictionary of Islam ( ${ }^{\circ}$ ed., p. 108). The Scarecrow Press, Inc.: Maryland. --- (2009c). JIHAD. In J. Woronoff (Ed.), Historical Dictionary of Islam2 ( $2^{\circ}$ ed., p. 169). The Scarecrow Press, Inc.: Maryland.

--- (2009d). RIDDAH. In J. Woronoff (Ed.), Historical Dictionary of Islam (p. 268). The Scarecrow Press, Inc.: Maryland.

BRAMON, D. (2009). Una introducción al islam: historia, religión y cultura. Crítica: Barcelona.

BRESC, H.; GUICHARD, P. \& MANTRAN, R. (2001). Europa y el Islam en la Edad Media. Crítica: Barcelona.

COSMA, G. (1940). De “Oeconomia” Incarnationis secundum S. Sophronium Hierosolymitanum. Urbaniana: Roma.

DURÁN VELASCO, J. F. (2012). El Islam frente a las otras religiones. Almuzara: España.

FLETCHER, R. (2005). La cruz y la media luna. Las dramáticas relaciones entre el cristianismo y el islam desde Mahoma hasta Isabel la Católica. Península: Barcelona.

FLORI, J. (2010). El islam y el fin de los tiempos. Akal: Madrid.

HOWARD-JOHNSTON, J. (1999) Historical Background. The Armenian History attributed to Sebeos. Liverpool University Press: Liverpool.

JANOSIK, D. (2003). John of Damascus' Response to the Islamic View of Justification by Works. Retrieved from http://www.phc.edu/UserFiles/ File/_Other Projects/Global Journal/10-1/Daniel_Janosik_John_of_ Damascus_Salvation_vol_10_no_1.pdf

KENNEDY, H. (2007). Las grandes conquistas árabes. Crítica: Barcelona.

MARÍN, J. (1997). Notas para una periodificación de la historia bizantina. Byzantion Nea Hellás, 16, 219 - 233.

MARTÍNEZ, F. J. (1985). Eastern christian apocalyptic in the early muslim period: Pseudo-Methodius and Psudo-Athanasius. Catholic University of America.

MELO, D. (2006). "Algunos aspectos en torno a la guerra en el Corán". ATUCSC, $45-58$.

RHODES, D. B. (2007). John Damascene in context: an examination of "The heresy of the ishmaelites" with special consideration given to the religious, political, and social contexts during the seventh and eighth century arab conquests. Virginia University of Lynchburg.

SÉNAC, P. (2011). El occidente medieval frente al islam. La imagen del otro. Editorial Universidad de Granada: Granada. 
SHAHID, I. \& BOSWORTH, C. E. (1997). Saracens. In The Encyclopaedia of Islam (Vol. IX, pp. 27 - 28). Brill: Leiden.

SUBHANI, Y. (1989). Luz de la eternidad. Vida del Profeta Muhammad (B.P.) e historia de los orígenes del Islam. Mezquita at-Tauhid: Buenos Aires.

TYAN, E. (1991). Djihad. In The Encyclopaedia of Islam (Vol. II). Brill: Leiden.

UBIERNA, P. (2000). La "leyenda del Último Emperador" en Bizancio y en el Cercano Oriente cristiano (ss. VII-X). Una instrumentalización del género apocalíptico. En G. Hansen (Ed.), Los caminos inexhauribles de la Palabra (Las lecturas creativas en la Biblia y de la Biblia). Lumen ISEDET: Buenos Aires. 